\title{
A multivariate technique for quantitative assessment of the opiate abstinence syndrome
}

\author{
LARRY P. GONZALEZ and HAROLD L. ALTSHULER \\ Neuropsychopharmacology Research Section, Texas Research Institute of Mental Sciences \\ and \\ Department of Pharmacology, Baylor College of Medicine, \\ Texas Medical Center, Houston, Texas 77030
}

\begin{abstract}
A procedure is described for the quantitative assessment of opiate abstinence. Subjects received exposure to either implanted placebo pellets or to morphine pellets implanted 1, 2, or 3 days. The naloxone-precipitated abstinence syndrome was observed in these animals, and a discriminant function was obtained which significantly described group differences on the multivariate profile of withdrawal responses. The function provided an accurate assessment of the length of morphine exposure in another independent set of subjects. The results suggest that discriminant function analysis is a useful procedure for assessing the opiate abstinence syndrome.
\end{abstract}

Many investigators have attempted to quantify the intensity of the opiate abstinence syndrome and, thus, the degree of physical dependence which results from exposure to opiate agonists. Early studies (Adler, Lin, Smith, Tresky, \& Gildenberg, 1974; Akera \& Brody, 1968; Cicero \& Meyer, 1973; Francis \& Schneider, 1971; Goode, 1971; Tilson, Rech, \& Stolman, 1973; Wikler \& Pescor, 1967) used singleresponse measures to assess physical dependence. Because of the possible dissociation of a single response from the total abstinence syndrome (Blasig, Herz, Reinhold, \& Zeiglgansberger, 1973; Collier, Francis, \& Schneider, 1972; Wei, 1973; Wei, Loh, $\&$ Way, 1973), the validity of the single-response measure has been questioned. Further, large between-subject variability may sometimes interfere with the reliability of single-response measures of opiate abstinence.

Recent attempts to quantify the severity of the abstinence syndrome use rating systems that grade the occurrence of several responses. These systems have not been uniform and there is disagreement over the importance of different abstinence signs. Wet-dog shakes, for example, receive a low score in Buckett's rating system (Buckett, 1964) and in that of Lorenzetti and Sancilio (1970), but a high score in that reported by Wei (1973). Kerr and Pozuelo (1971) found wet-dog shakes too unreliable a measure to include in their grading system.

In this paper we describe the use of discriminant function analysis to provide a set of weighting coefficients which statistically maximize the contribution of each of several variables in the quantitative assessment of the opiate abstinence syndrome. The procedure is a means for quantifying a multivariate profile, reducing intersubject variability and improving the reliability of assessment.

Discriminant function analysis is a technique for classifying subjects into groups by estimating each subject's position on a line that best separates the various groups (Cooley \& Lohnes, 1962). The estimated position is a linear function of the subject's scores on a set of response variables. In its application to assessing opiate abstinence, the line of separation is a line that discriminates between groups of subjects whose degree of physical dependence on morphine is different. The intensity of the withdrawal syndrome of an individual subject or of a treatment group may be assessed in terms of that subject's or group's relative position along the line of separation.

In the report which follows, a line of separation is determined for groups of subjects receiving different lengths of exposure to implanted morphine pellets. The line is then used to assess the abstinence syndrome in an independent set of subjects.

\section{METHODS}

\section{Subjects}

Ninety-six male Sprague-Dawley rats were used as subjects. All were 90 to 120 days old, weighing 200 to $250 \mathrm{~g}$. The animals were maintained under the same conditions of environment, diet, and handling for at least 5 days before treatment. They were housed in individual cages with free access to food and water throughout the study.

\footnotetext{
Procedure

The experimental procedures here are similar to those described previously (Gonzalez \& Altshuler, 1975) and will be reported only briefly here. Physical dependence on morphine was induced through subcutaneous (SC) implantation of morphine pellets
} 
containing $75 \mathrm{mg}$ morphine base. The development of physical dependence was assessed in terms of the withdrawal syndrome precipitated by injection of the morphine antagonist naloxone $\mathrm{HCl}, 0.4 \mathrm{mg} / \mathrm{kg}$, SC.

The abstinence syndrome was precipitated in subjects receiving morphine pellets 1,2 , or 3 days after pellet implantation. The response of morphine-naive subjects to naloxone was also obtained by injecting naloxone 1 day after the implantation of placebo pellets (containing no morphine) in some subjects.

Animals were observed individually in observation cages of stainless steel wire mesh, open at the top, measuring $43 \mathrm{~cm}$ long $\times 25 \mathrm{~cm}$ wide $\times 18 \mathrm{~cm}$ high. The frequency of occurrence of the following signs was recorded during the $30 \mathrm{~min}$ following naloxone injection: crossings from one quadrant of the observation chamber to another (activity counts), escape attempts (climbing on to the edge of the observation chamber, jumping on to the edge, or jumping out of the chamber), teeth-chattering episodes, and wet-dog shakes. Change in body weight during this period was also recorded.

Data were obtained from two sets of subjects, each set consisting of 48 rats: 12 placebo-implanted animals, 12 morphineimplanted animals which had naloxone injected 1 day after pellet implant, 12 morphine-implanted animals tested 2 days after implant, and 12 morphine-implanted animals tested 3 days after implant.

\begin{abstract}
Data Analysis
Multivariate analysis of variance was used to determine the significance of differences between groups of subjects exposed to placebo pellets or to morphine pellets for different lengths of time. The response measures of the first subject set were used in a discriminant function analysis to determine the linear function that describes the best line of separation for the four groups within that set, and the position of each group along this line was determined. The function was also used to compute the position of the four groups of the second subject set along the same line of separation. A univariate analysis of variance was used to determine whether groups from the two subject sets which received equal exposure to morphine differed significantly in position along the line of separation.
\end{abstract}

\section{RESULTS}

As determined by a multivariate analysis of variance, the length of exposure to morphine pellets significantly affected the response profiles of both sets of subjects $(p<.0001)$. All subjects lost weight following naloxone injection; longer morphine exposure was accompanied by greater weight loss. Responding on each of the other variables increased with morphine exposure. Post hoc comparisons of group differences on the univariate measures were performed with Duncan's multiple range test. Groups receiving different exposure to morphine were significantly different $(p<.01)$ from one another, with the exception of groups exposed to morphine pellets for 2 or 3 days. These were significantly different from the other groups, but not from one another $(p>.05)$. Response means for the two sets of subjects are presented in Tables 1 and 2 .

A discriminant function analysis of the data obtained from the first set of subjects produced the following equation, or discriminant function:

\section{Discriminant Function Score $=$}

$a_{1}$ (weight change) $+a_{2}$ (number of escape attempts) $+a_{3}$ (number of wet-dog shakes) $+a_{4}$ (number of teeth-chattering episodes) $+a_{5}$ (number of activity counts),

where $a_{1}=-.3117, a_{2}=-.0548, a_{3}=.1283$, $a_{4}=.0364$, and, $a_{5}=.0769$. Figure 1 presents the discriminant function scores obtained for each group of subjects within the first subject set and compares the scores obtained by using the data of the second set of subjects in the equation based on the first set. An analysis of variance indicates that the two sets of scores do not differ significantly $(p>.05)$ from one another. Further analysis of the discriminant function scores confirms the result obtained with univariate measures that groups with different exposure to morphine are significantly different $(p<.001)$ from one another, with the exception of differences between groups exposed to morphine for 2 or 3 days.

Table 1

Response Means ( \pm SEM) for Subject Set 1

\begin{tabular}{lcrrrr}
\hline Pellet Exposure & $\begin{array}{c}\text { Weight Change } \\
\text { (Grams) }\end{array}$ & $\begin{array}{c}\text { Escape } \\
\text { Attempts }\end{array}$ & $\begin{array}{c}\text { Wet-Dog } \\
\text { Shakes }\end{array}$ & $\begin{array}{c}\text { Teeth-Chattering } \\
\text { Episodes }\end{array}$ & $\begin{array}{c}\text { Activity } \\
\text { Counts }\end{array}$ \\
\hline Placebo & $-3.1 \pm 0.6$ & $0.1 \pm 0.1$ & $0.0 \pm 0.1$ & $1.0 \pm 0.7$ & $3.4 \pm 1.2$ \\
Morphine-1 day & $-11.8 \pm 1.1$ & $5.5 \pm 2.5$ & $6.8 \pm 1.3$ & $48.3 \pm 8.0$ & $24.3 \pm 2.2$ \\
Morphine-2 days & $-12.6 \pm 0.6$ & $12.3 \pm 5.2$ & $20.2 \pm 3.1$ & $47.3 \pm 5.1$ & $34.0 \pm 3.2$ \\
Morphine-3 days & $-14.2 \pm 1.1$ & $4.0 \pm 1.7$ & $18.0 \pm 1.8$ & $32.1 \pm 4.3$ & $36.0 \pm 5.2$ \\
\hline
\end{tabular}

Table 2

Response Means ( \pm SEM) for Subject Set 2

\begin{tabular}{lcccrrr}
\hline Pellet Exposure & $\begin{array}{c}\text { Weight Change } \\
\text { (Grams) }\end{array}$ & $\begin{array}{c}\text { Escape } \\
\text { Attempts }\end{array}$ & $\begin{array}{c}\text { Wet-Dog } \\
\text { Shakes }\end{array}$ & $\begin{array}{c}\text { Teeth-Chattering } \\
\text { Episodes }\end{array}$ & $\begin{array}{c}\text { Activity } \\
\text { Counts }\end{array}$ \\
\hline Placebo & $-3.7 \pm 0.9$ & $0.0 \pm 0.0$ & $0.0 \pm 0.0$ & $0.3 \pm 0.2$ & $0.7 \pm 0.4$ \\
Morphine-1 day & $-14.0 \pm 1.3$ & $0.5 \pm 0.3$ & $3.5 \pm 0.6$ & $30.0 \pm 5.1$ & $22.7 \pm 3.7$ & $3.7 \pm 0.2$ \\
Morphine-2 days & $-16.3 \pm 1.4$ & $4.3 \pm 1.6$ & $7.6 \pm 3.0$ & $43.0 \pm 10.5$ & 36.2 \\
Morphine-3 days & $-17.7 \pm 1.9$ & $6.5 \pm 3.0$ & $10.5 \pm 3.0$ & $48.8 \pm 10.4$ & $35.3 \pm 5.5$ \\
\hline
\end{tabular}




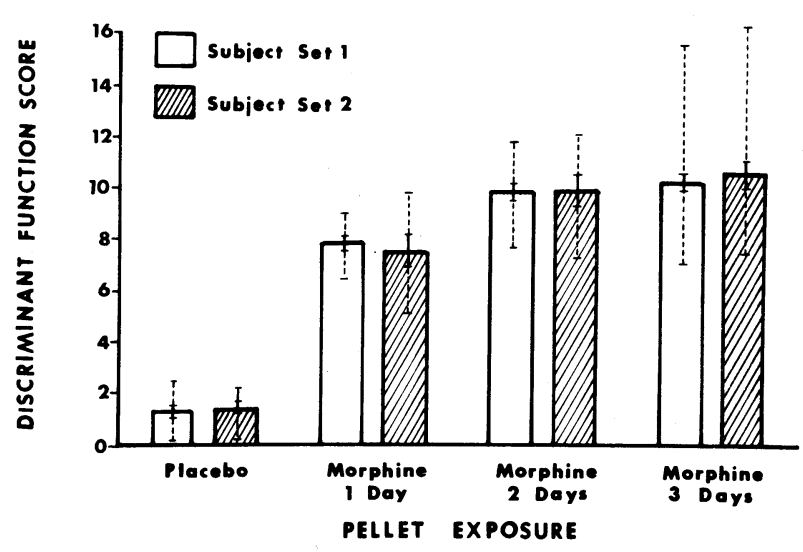

Figure 1. Discriminant function scores for subjects receiving different exposure to morphine pellets. The discriminant function, presented in the text, is based on Subject Set 1 , and the scores for this set are the standard scores. Solid bars indicate \pm S.E.M.; broken bars indicate the range of scores.

\section{DISCUSSION}

We considered the problem of assessing the intensity of the opiate abstinence syndrome of a morphine-dependent subject analogous to classifying that subject into a group of other subjects that have exhibited abstinence syndromes of similar intensity. We assumed that animals receiving equal exposure to morphine pellets should show abstinence syndromes of equal intensities. Such similarities and differences were evident in some single variables observed in this study for one set of subjects. Animals in both sets of subjects, for example, showed greater weight loss with longer exposure to morphine. The changes in weight noted among the groups in the first set of subjects could not, however, be used alone to predict the length of morphine exposure of a group in the second set. This was true for the other variables as well; single-response variables did not provide a reliable assessment of the abstinence syndrome.

With discriminant function analysis, the total response profile was transformed into a single discriminant function score that maximized the differentiation of groups receiving different lengths of morphine exposure. The discriminant function obtained from one set of subjects differentiated between groups receiving different lengths of morphine exposure and provided an accurate assessment of the abstinence syndrome in a second independent set of subjects (Figure 1).

In a typical application of this procedure, a discriminant function representing different intensities of the abstinence syndrome can be obtained for several groups of subjects. This can be done for subjects showing different degrees of physical de- pendence, as reported here, or by precipitating abstinence with different doses of a narcotic antagonist, as done by Wei (1973). Any number of variables and groups may be used to obtain the discriminant function, but if the design includes many variables and groups, more than one function may be necessary to significantly differentiate all of the groups. Computer programs are generally available for performing discriminant function analysis; the program "NYBMUL" of the computing center of the State University of New York at Buffalo was used in this study. Once calculated, the standard curve of discriminant function scores, or the line of separation, can be used to rate the intensity of the abstinence syndrome in other subjects or groups by comparing their discriminant function scores with the line of separation.

In this study, the line of separation of the various groups represents differences in the independent variable, length of exposure to implanted morphine pellets. Discriminant function analysis might also be applied in other experimental situations in which it is necessary to assess changes in a multivariate response profile. In any case, the line of separation represents differences in the independent variable. The procedure might be useful, for example, to assess drug tolerance, especially when several responses are used to measure pain thresholds (Yaksh \& Rudy, 1976), or to assess stereotypic behavior that is based on several response measures (Randrup \& Munkvad, 1975).

Discriminant function analysis combines the information from each variable in a multivariate response profile and increases assessment reliability. The technique has further advantages in that the discriminant function scores have the properties of an interval scale of measurement and are more likely to be normally distributed than single-response measures (Cooley \& Lohnes, 1962). Parametric tests can be applied, therefore, to the resulting scores to determine the significance of group differences. Discriminant function analysis as presented here was useful in assessing the response profile of the opiate abstinence syndrome.

\section{REFERENCES}

Adler, M. W., Lin, C., Smith, K. P., Tresky, R., \& GildenBERG, P. L. Lowered seizure threshold as a part of the narcotic abstinence syndrome in rats. Psychopharmacologia, 1974, 35, 243-247.

Akera, T., \& Brody, T. M. The addiction cycle to narcotics in the rat and its relation to catecholamines. Biochemical Pharmacology, 1968, 17, 675-688.

Blasig, J., Herz, A., Reinhold, K., \& Zieglgansberger, S. Development of physical dependence on morphine in respect to time and dosage and quantification of the precipitated withdrawal syndrome in rats. Psychopharmacologia, 1973, 33, 19-38. 
BuCKett, W. R. A new test for morphine-like physical dependence (addiction liability) in rats. Psychopharmacologia, 1964, 6, 410-416.

Cicero, T. J., \& Meyer, E. R. Morphine pellet implantation in rats: Quantitative assessment of tolerance and dependence. Journal of Pharmacology and Experimental Therapeutics, 1973, 184, 404-408.

Collier, H. O., Francis, D. L., \& Schneider, C. Modification of morphine withdrawal by drugs interacting with humoral mechanisms: Some contradictions and their interpretation. Nature, 1972, 237, 220-223.

COOLEY, W. W., \& LoHNEs, P. R. Multivariate procedures for the behavioral sciences. New York: Wiley, 1962.

Francis, D. L., \& SChNEIDER, C. Jumping after naloxone precipitated withdrawal of chronic morphine in the rat. British Journal of Pharmacology, 1971, 41, 424P-425P.

Gonzalez, L. P., \& Altshuler, H. L. Profiles of opiate abstinence: Rodent strain differences. Proceedings of the Society for Neuroscience, 1975, 1, 425.

Goode, P. G. An implanted reservoir of morphine solution for rapid induction of physical dependence in rats. British Journal of Pharmacology, 1971, 41, 558-566.

KerR, F. W. L., \& Pozuelo, J. Suppression of physical dependence and induction of hypersensitivity to morphine by stereotaxic hypothalamic lesions in addicted rats. Proceedings of the Mayo Clinic, 1971, 46, 653-665.
Lorenzetti, O. J., \& SAncilio, L. F. Morphine dependent rats as a model for evaluating potential addiction liability of analgesic compounds. Archives Internationales de Pharmacodynamie et de Therapie, 1970, 183, 391-402.

RANDRUP, A., \& MunKvad, I. Stereotyped behavior. Pharmacology and Therapy, Part B, 1975, 1, 757-768.

Tilson, H., Rech, R., \& Stolman, S. Hyperalgesia during withdrawal as a means of measuring the degree of dependence in morphine dependent rats. Psychopharmacologia, 1973, 28, 287-300.

WEI, E. Assessment of precipitated abstinence in morphinedependent rats. Psychopharmacologia, 1973, 28, 35-44.

WEI, E., LOH, H. H., \& WAY, E. L. Quantitative aspects of precipitated abstinence in morphine-dependent rats. Journal of Pharmacology and Experimental Therapeutics, 1973, 184, 398-403.

Wixler, A., \& Pescor, F. T. Classical conditioning of a morphine abstinence phenomenon, reinforcement of opioid-drinking behavior and "relapse" in morphine-addicted rats. Psychopharmacologia, 1967, 10, 255-284.

YAKSH, T. L., \& Rudy, T. A. Analgesia mediated by a direct spinal action of narcotics. Science, 1976, 192, 1357-1358.

(Received for publication February 16, 1977; accepted April 13, 1977.) 